\title{
ACCURATE BIT ERROR RATE EVALUATION IN OPTICALLY PREAMPLIFIED DIRECT-DETECTION
}

\author{
P. Martelli ${ }^{1,2}$, S. M. Pietralunga ${ }^{1}$, D. Nicodemi ${ }^{1}$, and M. Martinelli ${ }^{1,2}$ \\ ${ }^{1}$ CoreCom, Via G. Colombo 81, 20133 Milano, Italy, martelli@corecom.it \\ ${ }^{2}$ Politecnico di Milano, Dipartimento di Elettronica e Informazione, Via G. Ponzio 34/5, 20133 \\ Milano, Italy
}

\begin{abstract}
We present an accurate method for evaluating the bit error rate in optically preamplified direct-detection, by accounting for both the intersymbol-interference and the exact Laguerre photon-count statistics. A quantum-limited sensitivity of 33.9 photons/bit is derived.
\end{abstract}

\section{INTRODUCTION}

We propose a novel method to evaluate the bit error rate (BER) in optically preamplified intensity-modulated direct-detection (IM-DD) systems, based on the Laguerre photon-count statistics predicted by the theory of the photodetection $[1,2]$. The previously published works $[3,4]$ which use the Laguerre distribution to calculate the BER in on-off-keying (OOK) IM-DD systems, assume a measurement time $T_{m}=1 / R$, where $R$ is the bit rate, and do not consider the signal distortion due to the optical filtering, so that the intersymbolinterference (ISI) is neglected. Besides, they assume a number of modes $M$ of polarised ASE noise equal to the product between the optical bandwidth and the measurement time. These approximations can be considered as accurate whenever the optical bandwidth is much greater than $R$. Unfortunately, in dense wavelength-division multiplexing (DWDM) systems this condition is typically not verified, as one usually deals with optical and electrical bandwidths of comparable extent.

The method for evaluating the BER presented in this paper calculates the photon-count statistics of the directly detected noisy amplified signal, while accounting for both the effect of the ISI and the dependence of the ASE mode 
number on the optical filter shape and on the measurement time. The effects of optical filtering on the coherent part of the amplified signal and on the noise are separately considered.

\section{BIT ERROR RATE EVALUATION}

The scheme of the optically preamplified direct-detection receiver is shown in Fig. 1. An optical amplifier (OA) is followed by the cascade of a polariser (P), which eliminates the ASE component with state of polarisation orthogonal to the signal, an optical bandpass filter $(\mathrm{F})$, a photodetector (PD) and an electrical integrator (I) over $T_{m}$. At the quantum-limit the electrical signal obtained at the output of the photodetector is directly proportional to the photon-counts over $T_{m}$.

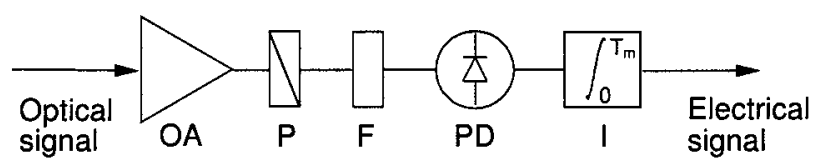

Figure 1. Scheme of an optically preamplified direct-detection receiver.

We consider an optical amplifier of gain $G$, with an input optical signal characterised by optical power $P_{\text {in }}(t)$, non-return to zero (NRZ) modulation format and rectangular intensity pulses. The optical field at the photodetector is given by the ASE noise superposed to a modulated coherent signal of power

$$
P_{s}(t)=\left|\sqrt{G P_{i n}(t)} * h(t)\right|^{2}
$$

where $h(t)$ is the baseband field impulse response of the optical filter and $*$ represents a convolution. In this way we account for the signal distortion due to the optical filter. According to the theory of the photodetection, the photoncount probability is described by the following Laguerre distribution:

$$
\begin{aligned}
p(n)= & \frac{\left(n_{A S E} / M\right)^{n}}{\left(1+n_{A S E} / M\right)^{n+M}} \cdot \exp \left(-\frac{n_{s}}{1+n_{A S E} / M}\right) \\
& \cdot L_{n}^{(M-1)}\left\{-\frac{n_{s}}{\left(1+n_{A S E} / M\right) \cdot n_{A S E} / M}\right\}
\end{aligned}
$$

where $L_{n}{ }^{(M-1)}$ is a generalised Laguerre polynomial [1]. The mean number of noise photon-counts is $n_{A S E}=n_{s p}(G-1) B_{e q} T_{m}$, where $B_{e q}$ is the noise equivalent bandwidth of the filter and $n_{s p}$ is the spontaneous emission factor [1]. The mean number $n_{s}$ of signal photon-counts, if a photodetector with unitary quantum efficiency is assumed, is calculated by integrating the optical power $P_{s}(t)$ over 
the measurement interval and dividing by the photon energy $h \nu$, in order to account for the effect of the ISI in terms of photon-counts. The exact number of modes $M$ is then given by the following integral [5]:

$$
M=\left\{\frac{1}{T_{m}} \int_{-T_{m}}^{T_{m}}\left(1-\frac{|\tau|}{T_{m}}\right) \cdot\left|g^{(1)}(\tau)\right|^{2} d \tau\right\}^{-1}
$$

The degree of first-order coherence $g^{(1)}(\tau)$ of the filtered ASE is the normalised autocorrelation of $h(t)$.

From the knowledge of both the optical filter spectral shape and $T_{m}$, it is possible to calculate the Laguerre photon-count distribution of Eq. (2) for any bit sequence. In the case of rectangular impulse response of the optical filter, with the condition $B_{e q} \geq R$, the photon-count probability in correspondence of a transmitted 'one' (or 'zero') is influenced only by the previous and subsequent bit. By assuming the bit sequences to be equiprobable, the BER results in:

$$
\mathrm{BER}=\frac{1}{2}\left[\sum_{n=0}^{n_{\text {th }}} \pi_{1}(n)+\sum_{n=n_{\text {th }}}^{\infty} \pi_{0}(n)\right]
$$

where $n_{t h}$ is the threshold level in terms of photon-counts and $\pi_{0}(n), \pi_{1}(n)$ are the photon-count probability distributions, in correspondence to a transmitted reference bit being respectively 'zero' and 'one'. These distributions are obtained by summing with same weighting factor the Laguerre photon-count distributions calculated in correspondence of all the bit sequences with respectively 'zero' and 'one' transmitted reference bit. After the calculation of the Laguerre photon-count probability distribution functions $p_{i j k}$ for each of threebit sequences $i j k$ (with $i, j, k=0,1$ ), the distributions $\pi_{0}, \pi_{l}$ can be determined as:

$$
\begin{aligned}
& \pi_{0}=\frac{1}{4} \sum_{i, k=0}^{1} p_{i 0 k} \\
& \pi_{1}=\frac{1}{4} \sum_{i, k=0}^{1} p_{i 1 k}
\end{aligned}
$$

The optimal threshold level is then given by the conditions $\pi_{1}\left(n_{t h}\right) \geq \pi_{0}\left(n_{t h}\right)$ and $\pi_{1}\left(n_{t h}-1\right) \leq \pi_{0}\left(n_{t h}-1\right)$. The BER obtained in such a way is a function of the noise equivalent optical bandwidth, of the measurement time and of the mean optical power at the amplifier input.

\section{NUMERICAL RESULTS}

By using the method previously described, we have numerically evaluated the BER at the quantum-limit in case of OOK-NRZ intensity modulation and 


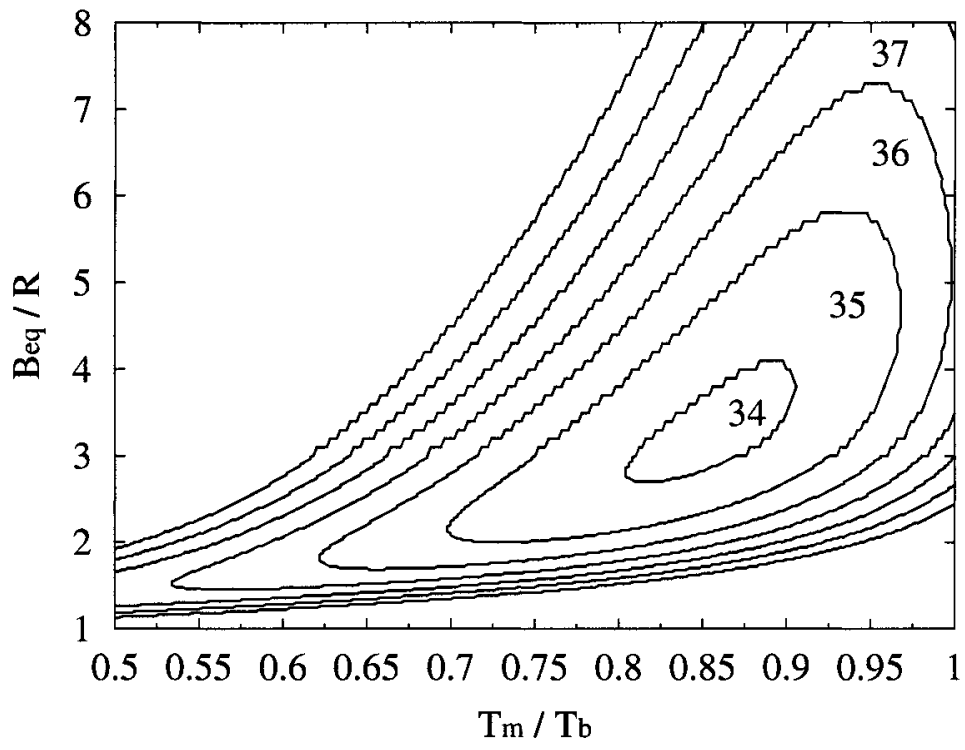

Figure 2. Contour lines of the quantum-limited sensitivity at BER $10^{-9}$ with rectangular $h(t)$, $G=30 \mathrm{~dB}$ and $n_{s p}=1$.

optically preamplified direct detection, with $G=30 \mathrm{~dB}$ and $n_{s p}=1$. The impulse response $h(t)$ of the optical filter is assumed to be rectangular.

Figure 2 shows the contour lines of the sensitivity calculated as a function of the noise equivalent bandwidth $B_{e q}$, normalised to the bit rate $R$, and of the measurement time $T_{m}$, normalised to the bit time $T_{b}=1 / R$. This sensitivity is defined as the mean photon number per bit at the amplifier input which gives a BER of $10^{-9}$. The optimal sensitivity is 33.9 photons per bit in correspondence of $B_{e q} / R=3.3$ and $T_{m} / T_{b}=0.86$.

In Figure 3 it is plotted the BER as a function of the ratio $B_{e q} / R$, in case of a mean vale of 34 photons/bit at the amplifier input and $T_{m} / T_{b}=0.86$. The continuous line represents the calculation with Laguerre distribution and exact value of $M$, given by Eq. (3). The dashed line corresponds to a Gaussian approximation of the photon-count distribution with exact $M$, while the dotted line corresponds to the case of Laguerre distribution with $M$ approximated by the product $B_{e q} T_{m}$. In all these cases, the effect of the ISI has been considered. The pronounced minimum of the BER as function of $B_{e q} / R$ is due to the competition between the reduction of ASE noise and the increase of the ISI, as $B_{e q} / R$ is reduced. It can be seen that within the Gaussian approximation, the BER is higher than the BER calculated by considering the Laguerre photoncount distribution; the discrepancy is appreciable near the optimum value for optical bandwidth that minimises the BER. Furthermore it is evident that the 


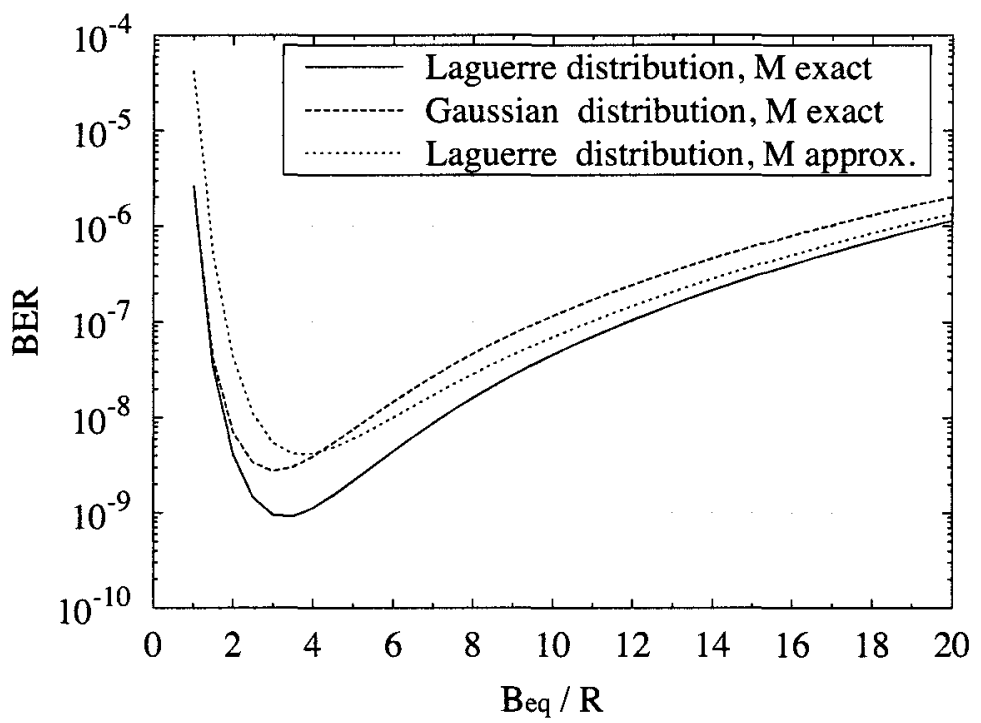

Figure 3. BER calculated as function of $B_{e q} / R$ for $T_{m} / T_{b}=0.86$ and 34 photons/bit at the amplifier input. Optical filter with rectangular $h(t), G=30 \mathrm{~dB}$ and $n_{s p}=1$.

approximation $M \simeq B_{e q} T_{m}$ gives a value of BER close to the exact one only at optical bandwidths much greater than the bit rate.

\section{CONCLUSIONS}

We have proposed a simple and accurate method to evaluate the performances of optically preamplified direct detection optical communications system in terms of BER calculation. Such a method is based on the Laguerre photon statistics and accounts for the exact noise mode number $M$ and for the effect of the ISI. Therefore, it is particularly suitable to model accurately the case of narrowband optical filtering, typical of DWDM systems, in which optical bandwidth and bit rate become comparable. In particular, in correspondence to optimised filtering condition, a quantum-limited optimal sensitivity of 33.9 photons per bit has been evaluated.

\section{REFERENCES}

[1] E. Desurvire, Erbium-doped fiber amplifiers - Principles and applications, J. Wiley \& Sons, 1994.

[2] B. E. A. Saleh, Photoelectron statistics, Springer-Verlag, 1978.

[3] T. Li and M. C. Teich, "Bit-error rate for a lightwave communication system incorporating an erbium-doped fibre amplifier," Electronics Letters, vol. 27, pp. 598-599, 1991. 
[4] H. A. Haus, Electromagnetic noise and quantum optical measurements, Springer-Verlag, 2000.

[5] J. W. Goodman, Statistical optics, J. Wiley \& Sons, 1985. 